\title{
Efficiency of Broadband Four-Wave Mixing Wavelength Conversion Using Semiconductor Traveling-Wave Amplifiers
}

\author{
Jianhui Zhou, Namkyoo Park, Jay W. Dawson, Kerry J. Vahala, Member, IEEE, \\ Michael A. Newkirk, Member, IEEE, and Barry I. Miller, Member, IEEE
}

\begin{abstract}
We present a theoretical analysis and experimental measurements of broadband optical wavelength conversion by four-wave mixing in semiconductor traveling-wave amplifiers. In the theoretical analysis, we obtain an analytical expression for the conversion efficiency. In the experiments, both up and downconversion efficiencies are measured as a function of wavelength shift for shifts up to $27 \mathrm{~nm}$. The experimental data are well explained by the theoretical calculation. The observed higher conversion efficiency for wavelength down-conversion is believed to be caused by phase interferences that exist between various mechanisms contributing to the four-wave mixing process.
\end{abstract}

\section{INTRODUCTION}

$\mathbf{O}$ PTICAL wavelength conversion devices are expected to play an important role in future broadband multichannel lightwave systems. Several techniques for wavelength conversion have been demonstrated. These include opticallytriggered multi-electrode DFB lasers [1], optical injectioninduced frequency shift in DFB lasers [2], gain saturation in semiconductor optical amplifiers [3], as well as nondegenerate four-wave mixing (FWM) in optical fibers [4], semiconductor lasers [5] and traveling-wave amplifiers [6].

Four-wave mixing in traveling-wave amplifiers (TWA's), when used for wavelength conversion, has several advantages. Owing to their traveling-wave operation, these devices offer the potential for very high speed operation as well as continuous tuning of the input and output wavelengths. In addition, since the converted signal is the phase-conjugate replica of the input signal, the conversion is transparent to the modulation format, and also provides the possibility for fiber-dispersioncompensation in lightwave communication systems.

In a typical FWM wavelength conversion experiment, pump and probe (input signal) waves are combined and coupled into a TWA. The beating of pump and probe fields in the TWA active layer induces dynamic gain and index gratings, and the subsequent scattering of the input fields from these gratings generates two sideband signals. The important contributing

Manuscript received July 13, 1993; revised November 1, 1993. This work was supported by the Office of Naval Research under contract N00014-911524 and by Northrop Corporation.

J. Zhou, N. Park, J. W. Dawson and K. J. Vahala are with the Department of Applied Physics, Mail Stop 128-95, California Institute of Technology, Pasadena, California 91125.

M. A. Newkirk and B. I. Miller are with AT\&T Bell Laboratories, Holmdel, New Jersey 07733.

IEEE Log Number 9214736 mechanisms are carrier density modulation caused by interband stimulated emission and intraband occupancy modulation via dynamic carrier heating and spectral hole burning. Owing to its ultrafast nature (relaxation times $<1 \mathrm{ps}$ ), intraband dynamics can play a dominant role in broadband wavelength conversion. This has been demonstrated in previous highly nondegenerate FWM measurements [7]-[10].

In this paper, we present a theoretical analysis in which coupled-amplitude equations are solved and an explicit expression is obtained for the FWM conversion efficiency. We also present measurements of wavelength conversion efficiency in TWA's as a function of wavelength shift $\Delta \lambda$ for shifts up to $27 \mathrm{~nm}$.

In the copropagation FWM geometry, guided waves in the TWA are given by $E_{j}(z) \exp \left[i\left(k_{j} z-\omega_{j} t\right)\right]$, where $j=$ $p, q, s$ indicate pump, probe and converted signal, respectively; $\left\{E_{j}(z)\right\}$ are the field amplitudes; and $z$ is the longitudinal coordinate along the propagation direction. The influence of interband and intraband mixing of pump and probe waves on the propagation of the three waves can be modeled using coupled-amplitude equations. Following procedures similar to those used in Agrawal's treatment [11], we obtain the following set of coupled equations

$$
\begin{aligned}
\frac{d E_{p, q}(z)}{d z}= & \frac{1}{2}\left(\frac{g_{0}}{1+\frac{P(z)}{P_{s}}}(1-i \alpha)-\alpha_{l}\right) E_{p, q}(z) \\
\frac{d E_{s}(z)}{d z}= & \frac{1}{2}\left(\frac{g_{0}}{1+\frac{P(z)}{P_{s}}}(1-i \alpha)-\alpha_{l}\right) \\
& \times E_{s}(z)-\kappa(z) E_{p}^{2}(z) E_{q}^{*}(z) \exp (i \Delta k z)
\end{aligned}
$$

where we have introduced the wave-number mismatch $\Delta k=$ $2 k_{p}-k_{q}-k_{s}$ and a four-wave mixing coupling coefficient $\kappa(z)$. In addition, $g_{0}$ is the unsaturated optical gain per unit length, with its wavelength dependence negligible for the devices tested. $P(z)$ is the total optical power at position $z$ in the waveguide, $P_{s}$ is the TWA's saturation power, $\alpha$ is the linewidth enhancement factor and $\alpha_{l}$ is the TWA's nonsaturable internal loss per unit length. In deriving Eq. (1), we have neglected the power that is coupled to pump and probe waves from neighboring wavelengths, since, for broadband wavelength conversion, the power transfer is negligible in comparison to the power in the pump and probe, themselves. 
The coupling coefficient $\kappa(z)$ is central to this theoretical analysis. To simplify the analysis, we treat contributing mechanisms to FWM-carrier density modulation, dynamic carrier heating and spectral hole burning-as independent processes. This is justified, in part, by noting that damping effects associated with these mechanisms are caused by interactions with independent baths. By evaluating the polarizations induced by the above mechanisms at the signal wavelength, $\kappa(z)$ is found to be

$$
\kappa(z)=\frac{1}{2} \frac{g_{0}}{1+\frac{P(z)}{P_{s}}} \sum_{m=1}^{3} \frac{1-i \alpha_{m}}{1-i 2 \pi f \tau_{m}} \cdot \frac{1}{P_{m}}
$$

where $m=1,2,3$, for carrier density modulation, dynamic carrier heating and spectral hole burning, respectively. $f$ is the detuning frequency, defined as the difference between the optical frequencies of the pump and probe waves; $\left\{\tau_{m}\right\}$ and $\left\{P_{m}\right\}$ are the lifetimes and saturation powers associated with the mechanisms; and $\left\{\alpha_{m}\right\}$ give the ratio between the real and the imaginary parts of the refractive index change induced by the mechanisms.

Using the boundary condition $E_{s}(0)=0$, we solve Eqs. (1) and (2) and find that under moderate TWA saturation the converted signal amplitude at the TWA output $(z=l)$ can be expressed by

$$
E_{s}(l)=-\frac{E_{p}^{2}(l) E_{q}^{*}(l) \kappa(l) l e^{i \Delta k l}}{0.23 G+i \Delta k l}
$$

where $G=4.34 \int_{0}^{l}\left(\frac{g_{0}}{1+\frac{P(z)}{P_{l}}}-\alpha_{l}\right) d z$ is the TWA saturated gain in $\mathrm{dB}$. The phase mismatch term can be expressed as $\Delta k l=\frac{\pi}{2} \cdot \frac{d n_{g}}{d \lambda} \cdot\left(\frac{\Delta \lambda}{\lambda}\right)^{2} \cdot l$, where $\frac{d n_{g}}{d \lambda}$ is the group index dispersion. Using $\frac{d n_{g}}{d \lambda} \sim-0.7 \mu \mathrm{m}^{-1}$, measured by Hall $e$ t al. [12], and $l=0.8 \mathrm{~mm}$ for the devices in this study, the phase mismatch for $\Delta \lambda=27 \mathrm{~nm}$ is estimated to be -0.27 , which is negligible in comparison to the first term for a typical optical gain of $G \sim 15 \mathrm{~dB}$.

Using Eq. (4), the conversion efficiency, defined as the ratio between the converted signal power and the input probe power, is given in $\mathrm{dB}$ by

$$
\begin{aligned}
\eta & =10 \log \frac{P_{s}(l)}{P_{q}(0)} \\
& =3 G+2 I_{p}+20 \log \left|\sum_{m=1}^{3} c_{m} \cdot \frac{1}{1-i 2 \pi f \tau_{m}}\right|
\end{aligned}
$$

where $I_{p}$ is the input pump power in $\mathrm{dBm}$ and where the complex coupling coefficient, $c_{m}$, is defined as

$$
c_{m}=\frac{g_{0} l}{0.46 G\left(1+\frac{P(l)}{P_{s}}\right)} \cdot \frac{1-i \alpha_{m}}{P_{m}}
$$

We note that the term $3 G$ is present in Eq. (5), which results, physically, from the fact that both the pump and probe waves experience an optical gain of $G$ (note that $G$ affects the pump wave twice due to the form of Eq. (4)). TWA's with large optical gain are therefore critical to efficient wavelength conversion.

The experimental arrangement for this study was similar to what we have described in Ref. [9]. The TWA's used

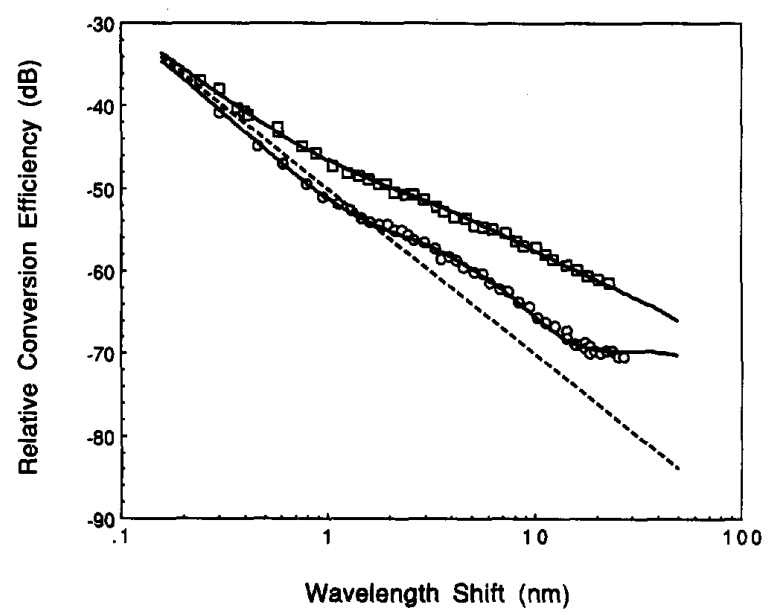

Fig. 1. Measured wavelength conversion efficiency relative to $3 G+2 I_{p}$ versus up-conversion (circle), and down-conversion (square) wavelength shifts, showing theoreticalfit (solid line) and $20 \mathrm{~dB} / \mathrm{dec}$ line (dashed line). Device tested is a tensile-strained TWA biased at $100 \mathrm{~mA}$.

in the measurements were compressively and tensile-strained InGaAs/InGaAsP multiple quantum well devices operating at $1.5 \mu \mathrm{m}$. Details on these devices appear in Refs. [13] and [14]. Three, single-frequency, tunable, Er-doped fiber ring lasers [15] were used as pump, probe, and local oscillators in the measurement. The converted signal was heterodyne detected by mixing the signal with the fiber-laser local oscillator output and tuning the local oscillator relative to the signal frequency to provide an IF signal. The IF mixing frequency was maintained at $4.00 \mathrm{GHz}$ throughout the measurements to eliminate the frequency dependence of the detection electronics. The detected photocurrent was measured using an electronic spectrum analyzer, and the input wavelengths and the optical powers of pump and probe were measured using an optical spectrum analyzer.

Using this experimental setup, both the up and downconverted signals were measured, and both compressively and tensile-strained TWA's were tested. The maximum wavelength conversion spacing measured was as large as $27 \mathrm{~nm}$. The conversion efficiency relative to $3 G+2 I_{p}$ was then evaluated as a function of the wavelength shift. Shown in Fig. 1 are the data from the tensile-strained device and the theoretical fit based on Eq. (5).

As can be seen in Fig. 1, Eq. (5) provides an excellent fit for both up and down-conversion efficiency spectra. The fitting parameters are as follows: $\tau_{1}=200 \mathrm{ps}, \tau_{2}=650$ fs, $\tau_{3}=50 \mathrm{fs}, C_{1}=0.24 e^{-i 1.30}, C_{2}=0.0027 e^{i 1.30}$, $C_{3}=0.00048 e^{i 1.53}$. The efficiency asymmetry with respect to up and down-conversion is believed to be caused by the phase interferences which occur between the various contributing mechanisms. The down-conversion shows overall higher efficiency because of constructive phase interferences which exist on this detuning side. The compressively-strained device data, shown in Fig. 2, also exhibit similar features. This fit is obtained using the same relaxation time constants as for 


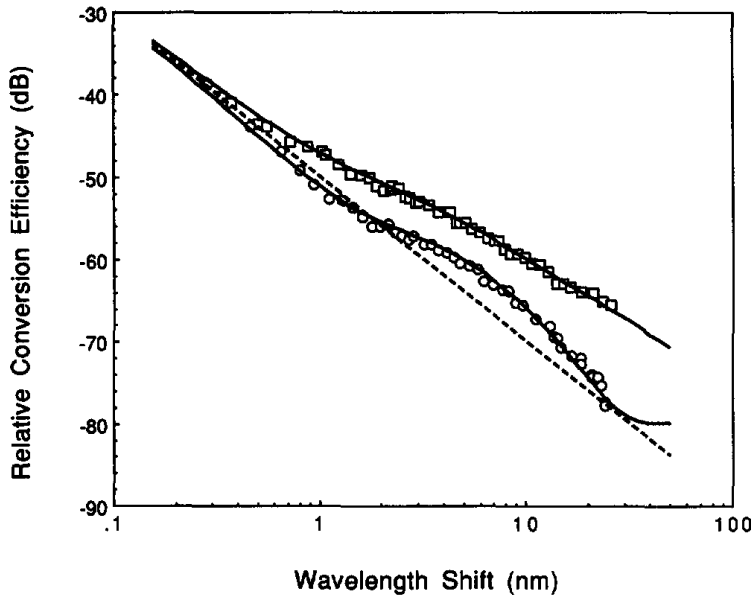

Fig. 2. Measured wavelength conversion efficiency relative to $3 G+2 I_{p}$ versus up-conversion (circle), and down-conversion (square) wavelength shifts, showing theoreticalfit (solid line) and $20 \mathrm{~dB} / \mathrm{dec}$ line (dashed line). Device tested is a compressively-strained TWA biased at $150 \mathrm{~mA}$.

the tensile device, but different complex coupling coefficients: $C_{1}=0.24 e^{-i 1.30}, C_{2}=0.0027 e^{i 1.42}, C_{3}=0.00023 e^{i 3.13}$. Interpretation of these fitting parameters can be found in Ref[10].

The relative efficiency spectra measured in this study should be useful for the design of four-wave mixing wavelength converters. They make it possible to quickly estimate the actual conversion efficiency at any $\Delta \lambda$-shift provided that the optical gain and input pump power are known. For example, in the case of tensile TWA measurements reported here, the saturated TWA gain was $\sim 15 \mathrm{~dB}$ and the input pump power was $\sim-9$ $\mathrm{dBm}$, giving a conversion efficiency of $-30 \mathrm{~dB}$ for $\Delta \lambda=10$ $\mathrm{nm}$ (up-conversion). If the saturated optical gain could be increased to $25 \mathrm{~dB}$ while other parameters remain unchanged, the efficiency would become $0 \mathrm{~dB}$ or $100 \%$. This illustrates the importance of TWA gain for efficient wavelength conversion. Additionally, the measured efficiency spectra indicate the presence of ultrafast intraband processes in the FWM process, and can provide insight into the ultrafast dynamics of the semiconductor active layers. We have discussed broadband FWM wavelength conversion as a spectroscopic tool in a recent paper [10] and the FWM mechanism assignments used here result, in part, from that work.

In conclusion, we have measured the FWM conversion efficiency versus wavelength shift for shifts up to $27 \mathrm{~nm}$. The efficiency spectra were well explained by a theoretical analysis which incorporates the contributions to the conversion process from carrier density modulation, dynamic carrier heating and spectral hole burning. The theoretical results and the measured efficiency spectra presented in this paper should be of practical importance for design and analysis of four-wave mixing wavelength converters.

\section{REFERENCES}

[1] H. Kawaguchi, K. Magari, H. Yasaka, M. Fukada and K. Oe, "Tunable optical-wavelength conversion using an optically triggerable multielectrode distributed feedback laser diode," IEEE J. Quantum Electron., vol. 24, pp. 2153-2159, 1988.

[2] K. Inoue, "Wavelength conversion for frequency-modulated light using optical modulation to oscillation frequency of a DFB laser diode," IEEE J. Lightwave Technol., vol. 8, pp. 906-911, 1990.

[3] B. Glance, J. M. Wiesenfeld, U. Koren, A. H. Gnauck, H. M. Presby and A. Jourdan, "High performance optical wavelength shifter," Electron. Lett., vol. 28, pp. 1714-1715, 1992.

[4] $\mathbf{K}$. Inoue and $\mathbf{H}$. Toba, "Wavelength conversion experiment using fiber four-wave mixing," IEEE Photon. Technol. Lett., vol. 4, pp. 69-72, 1992.

[5] S. Murata, A. Tomita, J. Shimizu and A. Suzuki, "THz optical frequency conversion of $1 \mathrm{~Gb} / \mathrm{s}$ signal using highly nondegenerate four-wave mixing in an InGaAsP semiconductor laser," IEEE Photon. Technol. Lett., vol. 3, pp. 1021-1023, 1991.

[6] M. C. Tatham and G. Sherlock, "20 nm Wavelength conversion using ultrafast highly nondegenerate four-wave mixing," OSA Integ. Photon. Res. Topic Meeting, paper PD1, 1993.

[7] L. F. Tiemeijer, "Effects of nonlinear gain on four-wave mixing and asymmetric gain saturation in a semiconductor laser amplifier," Appl. Phys. Lett., vol. 59, pp. 499-501, 1991.

[8] K. Kikuchi, M. Kakui, C. E. Zah and T. P. Lee, "Observation of highly nondegenerate four-wave mixing in a $1.5 \mu \mathrm{m}$ traveling-wave semiconductor optical amplifier and estimation of nonlinear gain coefficient," IEEE J. Quantum Electron., vol. 28, pp. 151-156, 1992.

[9] J. Zhou, N. Park, J. W. Dawson, K. J. Vahala, M. A. Newkirk, U. Koren and B. I. Miller, "Highly nondegenerate four-wave mixing and gain nonlinearity in a strained multiple-quantum-well optical amplifier," Appl. Phys. Lett., vol. 62, pp. 2301-2303, 1993.

[10] J. Zhou, N. Park, J. W. Dawson, K. J. Vahala, M. A. Newkirk and B. I. Miller, "Terahertz four-wave mixing spectroscopy for study of ultrafast dynamics in a semiconductor optical amplifier," Appl. Phys. Lett., vol.63, pp. 1179-1181, 1993

[11] G. P. Agrawal, "Population pulsations and nondegenerate four-wave mixing in semiconductor lasers and amplifiers," J. Opt. Soc. Am. B, vol. 5, pp. 147-158, 1988.

[12] K. L. Hall, G. Lenz and E. P. Ippen, "Femtosecond time domain measurements of group velocity dispersion in diode lasers at $1.5 \mu \mathrm{m}$," IEEE J. Lightwave Technol, vol. 10, pp. 616-619, 1992.

[13] M. A. Newkirk, U. Koren, B. I. Miller, M. D. Chien, M. G. Young, T. L. Koch, G. Raybon, C. A. Burrus, B. Tell and K. F. Brown-Goebeler, "Three-section semiconductor optical amplifier for monitoring of optical gain," IEEE Photon. Technol. Lett., vol. 4, pp. 1258-1260, 1992.

[14] B. I. Miller, U. Koren, M. A. Newkirk, M. G. Young, R. M. Jopson, R. M. Derosier and M. D. Chien, "Tensile-strained InGaAs/nnGaAsP quantum-well optical amplifier with a wide spectral gain region at 1.55 $\mu \mathrm{m}$," IEEE Photon. Technol. Lett., vol. 5, pp. 520-522, 1993.

[15] N. Park, J. W. Dawson, K. J. Vahala and C. Miller, "All fiber, low threshold, widely tunable single-frequency, erbium-doped fiber ring laser with a tandem fiber Fabry-Perot filter," Appl. Phys. Lett., vol. 59, pp. 2369-2371, 1991. 